\title{
Diagnosing in the dark: atypical proteinuria etiology
}

\author{
Stephen Cornish (Meds 2015) and Eric Roszell (Meds 2016) \\ Faculty Reviewer: Dr. Faisal Rehman, MD, FRCPC, M.Ed (Department of Medicine, Division of Nephrology)
}

A zebra is a member of the species of African equids, of the horse family, whose appearance is defined by striking black and white stripes. These animals are rarely seen outside of their native continent, except for their requisite presence in zoos, menageries, and circuses. When we think of something that is common, a zebra may define the direct antithesis to our notion of commonality. Likewise, a zebra patient is, for a physician, a peculiar blend of an exciting presentation and a frustrating diagnosis. The helpful adage "common things are common" can be summarily thrown out of the nearest window when dealing with the contents of the Zebra Files.

Our case begins with an 80 year old gentleman presenting to his primary care physician with an acute history of leg swelling and urinary frequency over the past three weeks. An evaluation of the present complaint determined that he was also experiencing a reduced appetite, nausea and vomiting. On examination, the man's blood pressure was $138 / 78$, and the lower extremities demonstrated the features of pitting edema. Investigations were ordered, and returned as follows: blood urea nitrogen $(\mathrm{BUN})$ of $7.3 \mathrm{mmol} / \mathrm{L}$ (normal range $2.9-8.6 \mathrm{mmol} / \mathrm{L}$ ), serum creatinine $(\mathrm{Cr})$ of $457.6 \mathrm{umol} / \mathrm{L}$ (normal range $53-115 \mathrm{umol} / \mathrm{L}$ ), and a serum albumin level of $25 \mathrm{~g} / \mathrm{L}$ (normal range $35-50 \mathrm{~g} / \mathrm{L}$ ). Urinalysis was performed, which detected the presence of protein (graded as a $3+$ ) as well as glucose in the urine, with no remarkable microscopic findings. ${ }^{1}$ With these results and the clinical picture revealed, we can begin to hypothesize.

The finding of pitting edema on physical exam suggests an accumulation of fluid in the interstitial space of body tissues. Fluid typically pools in dependent areas of the body like the legs and feet. ${ }^{2}$ This is reflected in our patient, who had noticed an abnormal swelling of his lower limbs. Pitting edema is a sign of volume overload in patients, which results in excess fluid seeping out of the vasculature into the interstitial space of body tissues. There are several mechanisms by which this volume overload phenomenon can occur. For the sake of brevity, heart failure, liver diseases such as cirrhosis, renal sodium retention, nephrotic syndrome, drug-induced, and idiopathic edema are all potential culprits, especially in an individual of advanced age. ${ }^{3}$ Differentiating between these etiologies is critical in order to proceed.

Building on our understanding of this patient's volume status, we can examine the results of the initial investigations. Both urea and creatinine were found to be elevated, which indicate impairment of renal function. The key to the picture here is the urinalysis test. The significant presence of protein in the urine, classified in this case as proteinuria of 3 out of a possible 4 degree of severity, should not be seen in a healthy person and is an indicator of disease.

A differential diagnosis for heavy proteinuria would include glomerular causes such as glomerulonephritis, diabetic glomeruloscle- rosis, systemic lupus erythematosus, amyloidosis, and vasculitis. Other causes may include, monoclonal proteinuria, multiple myeloma, lymphoma, or other neoplasia. ${ }^{4}$ Renal function is dependent upon filtration in the glomerular unit, resulting in the removal of wastes and the retention of blood cells and proteins. Failure to retain proteins suggests damage to the glomerulus, the filtration unit of the kidney. The absence of blood in the urine, or hematuria, is also helpful. Heavy proteinuria without hematuria falls into one category of renal disease (nephrotic), while the combination is indicative of a different type of pathology (nephritic). ${ }^{5}$ The measurement of a low albumin in the investigation of this patient is reflective of the heavy proteinuria.

The patient was referred by his primary care physician to a nephrology clinic for further evaluation. Repeat urinalysis was performed. A 24 hour urine collection for protein was performed, which is more helpful for evaluating the amount of proteinuria than a dipstick urinalysis. The patient was losing $20.7 \mathrm{~g}$ of protein over a 24 hour period in his urine. This more focused analysis returned another curious result; in addition to the previously detected proteinuria, trace blood also appeared in the urine. Microscopic analysis identified 10-20 red blood cells per magnification field, with some granular casts. ${ }^{1}$ The patient has now demonstrated some degree of hematuria observed in the setting of heavy proteinuria.

The finding of hematuria shifts the diagnostic focus away from a nephrotic syndrome, which is categorically limited to protein wasting in the urine, and usually not blood. Glomerulonephritis is an inflammatory process defined by the presence of red blood cell casts or dysmorphic red blood cells in urine, moderate proteinuria, hypertension, and renal failure. ${ }^{6}$ Causes of the nephritic syndrome all relate to various defective autoimmune processes; damage from these circulating factors occurs progressively as these factors accumulate during glomerular filtration. The underlying cause of the glomerulonephritis can be determined by serological tests for these autoimmune factors.

Naturally, in our "Zebra" patient, the usual suspects in the nephritic syndrome (anti-nuclear antibodies, ANCA and anti-GBM antibodies, cryoglobulins, Hep C antibody, ASO titres) turned up negative. However, two proteins (C3 and C4) were found to be abnormally low. ${ }^{1}$ These are normally involved in the complement cascade vital to the body's immune response.

A renal biopsy was performed to determine the glomerular pathology. Light microscopic evaluation showed classic characteristics of a membranoproliferative glomerulonephritis, with formation of pathological crescents in $50 \%$ of glomeruli. A Congo red stain was performed on the biopsy sample; it returned with a negative result, ruling out renal amyloidosis. ${ }^{1}$ After a circuitous diagnostic route, the electron microscopy and immunofluorescence on the kidney biopsy specimen did provide an 


\section{ZEBRA FILES}

answer. The architecture of the glomerular basement membrane was being disrupted by randomly arranged fibrillary deposits. Final diagnosis was determined: fibrillary glomerulonephritis with crescent formation, in the presence of hypocomplementemia.

Fibrillary glomerulonephritis is an uncommon disease of the glomerulus leading to renal dysfunction. ${ }^{7}$ This dysfunction is caused by a buildup of randomly arranged fibrillar deposits in the glomerular base membrane and mesangium. ${ }^{8}$ The disease was first distinguished in 1977 from amyloidosis on the basis that congo red stains, which stain amyloid fibers, were negative in several patient's kidney biopsies. ${ }^{1}$ Fibrillary glomerulonephritis has been associated with different malignancies, monoclonal gammopathies, and autoimmune disorders. ${ }^{7}$ Despite the thorough characterization of fibrillary glomerulonephritis, the pathology of the disorder is not fully understood although it is known that the fibrillary deposits are derived from immunoglobulins. ${ }^{9}$

Crescents formation, as seen in our "zebra" patient, is seen in approximately $20 \%$ of cases of fibrillary glomerulonephritis. Crescents result from a nonspecific response to severe injury of the glomerular capillary wall. These crescents can involve anywhere from $10 \%$ to $80 \%$ of the glomerulus. ${ }^{1}$ Since crescents result from a nonspecific response, they can be seen in any type of severe glomerular disease.

Although fibrillary glomerulonephritis is not generally considered a rare disease, as it is present in approximately $1 \%$ of kidney biopsies, the added complication of hypocomplementemia has only been reported on one other occasion in the literature. ${ }^{7}$ Hypocomplentemia can refer to low levels of the molecular mediators of complement; in our "zebra" patient it refers to the decreased amount of $\mathrm{C} 3$ and $\mathrm{C} 4$.

The treatment of fibrillary glomerulonephritis with crescent formation and hypocomplementemia has not been well established due to the rarity of the condition. Unfortunately, many of the treatment options for other types of glomerulonephritis, such as steroids or cytotoxic agents, are ineffective on fibrillary glomerulonephritis. ${ }^{7}$ Conservative use of angiotensin inhibition is often used to control blood pressure and slow the progress of the disease. ${ }^{10}$ Some of the therapies that have been attempted to some success involve the use of glucocorticoids, prednisone, cytotoxic agents such as cyclophosphamide, and rituximab. ${ }^{11}$

The prognosis for patients with fibrillary glomerulonephritis is often grave due to the dearth of treatment options. The expected time course can be estimated at the time of a renal biopsy by different histological features seen under light microscopy. ${ }^{12}$ Approximately 50\% of patients will progress to end-stage renal disease within two to six years. Among the remaining patients, only $10 \%$ achieve complete or partial remission while $40 \%$ have progressive renal disease. ${ }^{10}$ With this outlook, our patient may have become one of the growing numbers of patients who are dependent on renal dialysis therapy, a difficult reality that defines the medical care of so many elderly patients.

\section{REFERENCES:}

1. Adey DB, MacPherson BR, Groggel GC. Glomerulonephritis with associated hypocomplementemia and crescents: an unusual case of fibrillary glomerulonephritis. J Am Soc Nephrol. 1995 Aug;6(2):171-176.

2. Humphreys MH. Mechanisms and management of nephrotic edema. Kidney Int. 1994 Jan;45 (1):266-281.

3. Cho S, Atwood JE. Peripheral edema. JAMA. 2002 Nov; 113(7):580-586.

4. Fine LG, Salehmoghaddam S. Clinical methods: the history, physical, and laboratory examinations. Boston, MA: Butterworths; 1990. 872-873 p.

5. Orth SR, Ritz E. The nephrotic syndrome. NEJM. 1998 Apr;338(17):12021211.

6. Dwyer JP, Lewis JB. Cecil essentials of medicine $8^{\text {th }}$ edition. Philadelphia,
PA: Saunders Elsevier. 324 p.

7. Ray S, Rouse K, Appis A, Novak R, Haller NA. Fibrillary glomerulonephritis with hepatitis $\mathrm{C}$ viral infection and hypocomplementemia. Ren Fail. 2008;30(7):759-762.

8. Javaid MM, Denley H, Tagboto S. Fibrillary glomerulonephritis with small fibrils in a patient with the antiphospholipid antibody syndrome successfully treated with immunosuppressive therapy. BMC Nephrol. 2007 May;8(1):7.

9. Schwartz MM, Korbet SM, Lewis EJ. Immunotactoid glomerulopathy. J Am Soc Nephrol. 2002 May;13(5):1390-1397.

10. Nasr SH, Valeri AM, Cornell LD, Fidler ME, Sethi S, Leung N, Fervenza FC. Fibrillary glomerulonephritis: a report of 66 cases from a single institution. Clin J Am Soc Nephrology. 2011 Apr;6(4):775-784.

11. Collins M, Navaneethan SD, Chung M, Sloand J, Goldman B, Appel G, Rovin BH. Rituximab treatment of fibrillary glomerulonephritis. Am J Kid Dis. 2008 Dec;52(6):1158-1162.

12. Rosenstock JL, Markowitz GS, Valeri AM, Sacchi G, Appel GB, D’Agati VD. Fibrillary and immunotactoid glomerulonephritis: distinct entities with different clinical and pathologic features. Kidney Int. 2003 Apr;63(4):1450-1461.

\section{Experience learning, living, and working in Southwestern Ontario.}

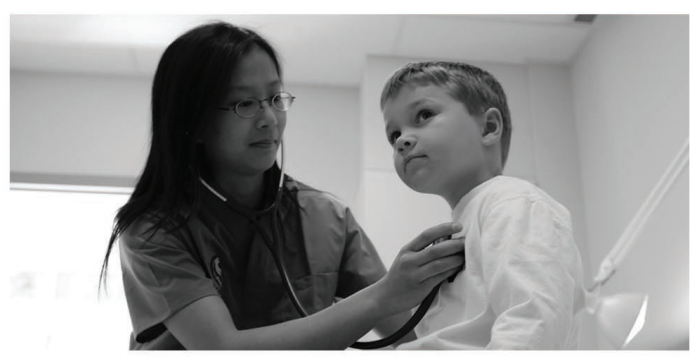

The Southwestern Ontario Medical Education Network (SWOMEN) at the Schulich School of Medicine \& Dentistry offers rural and regional medical training opportunities for undergraduate and postgraduate learners at more than 50 communities across Southwestern Ontario.

You will benefit from a low teacher-learner ratio and hands-on learning experience. You will also receive support for travel and accommodation for rotations of a minimum of four weeks in length.

Learn more by visiting www.schulich.uwo.ca/swomen

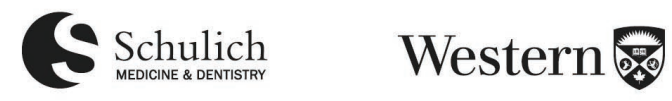

519.858.5152 or toll-free 1.877.237.9676 - SWOMEN@schulich.uwo.ca 